\title{
Interfaces
}

\section{Côme Martin. Lire le récit multimodal, à la limite de ses habitudes}

\section{Benoît Crucifix}

\section{(2) OpenEdition}

1 Journals

\section{Édition électronique}

URL : https://journals.openedition.org/interfaces/2970

DOI : 10.4000/interfaces.2970

ISSN : 2647-6754

Éditeur :

Université de Bourgogne, Université de Paris, College of the Holy Cross

\section{Édition imprimée}

Date de publication : 12 juillet 2021

ISSN : 1164-6225

\section{Référence électronique}

Benoît Crucifix, "Côme Martin. Lire le récit multimodal, à la limite de ses habitudes », Interfaces [En ligne], 45 | 2021, mis en ligne le 12 juillet 2021, consulté le 20 septembre 2021. URL : http:// journals.openedition.org/interfaces/2970; DOI : https://doi.org/10.4000/interfaces.2970

Ce document a été généré automatiquement le 20 septembre 2021.

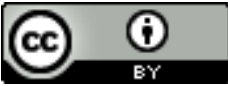

Les contenus de la revue Interfaces sont mis à disposition selon les termes de la Licence Creative Commons Attribution 4.0 International. 


\title{
Côme Martin. Lire le récit multimodal, à la limite de ses habitudes
}

\author{
Benoît Crucifix
}

\section{RÉFÉRENCE}

Côme Martin. Lire le récit multimodal, à la limite de ses habitudes. Liège : Presses universitaires de Liège (coll. « ACME »), 2020. 260 pages. ISBN 9782875622433.

1 Le format est souvent considéré comme un attribut de standardisation qui, en ce qu'il qualifie des usages normés, est imperceptiblement intégré au sein des pratiques et des expériences de lecture. Poussant le lecteur "à la limite de ses habitudes », certaines œuvres et objets peuvent soudain faire apparaître le format en tant que tel aux yeux de celui-ci. Ce sont ce type de productions et le moment de réflexivité matérielle qu'elles déclenchent dans la lecture, qui sont au cœur de l'ouvrage que Côme Martin a consacré au livre codex et à sa transformation au tournant du vingt-et-unième siècle. Version remaniée d'une thèse en littérature américaine contemporaine, Lire le récit multimodal aborde cette vaste question par le prisme de la multimodalité et des rapports texteimage.

2 Avec un tel point d'entrée, Côme Martin se joint à un concert d'études sur le roman multimodal et la littérature expérimentale ${ }^{1}$. La grande originalité de son travail est de resituer la bande dessinée contemporaine au sein de ces discussions qui, souvent, reconnaissent cette proximité pour mieux la mettre de côté. Lire le récit multimodal, au contraire, traite ensemble et d'un même geste roman graphique et roman multimodal, qui forment dans son étude deux faces d'une même pièce sans pour autant aplanir leurs différences historiques. Les récits numériques et les albums illustrés y sont également convoqués de façon ponctuelle, dans un comparatisme médiatique qui reste présent tout au long de ce travail. Martin saisit un moment particulier de l'histoire du livre aux États-Unis, au tournant du vingt-et-unième siècle, alors que le paysage médiatique est en pleine recomposition, et que les aménagements technologiques dans l'impression et 
l'édition rendent possible et démocratisent un usage ludique, innovant ou transgressif des formats standards.

3 L'ouvrage de Côme Martin décrit ainsi admirablement la redistribution des rapports entre format, texte et image dont témoignent cette synergie entre la part croissante du visuel dans le roman contemporain, l'investissement du support livresque par la bande dessinée (jusque-là avant tout publiée dans des journaux, périodiques, et fascicules), et l'influence des nouveaux médias numériques. Si l'auteur souligne d'autres proximités qu'il conviendrait d'élucider dans le cadre du récit multimodal (la littérature numérique et l'album illustré pour enfants), son parti pris n'a rien d'arbitraire et trouve une pertinence à la fois dans la proximité poétique des œuvres convoquées et dans leur paysage médiatique commun. Sans jamais oublier de situer les œuvres dans leurs traditions historiques, Côme Martin travaille avant tout par lecture rapprochée d'« œuvres-exemples » (8) volontairement choisies pour leur caractère exceptionnel. Le corpus se construit par saillance, le haut degré d'expérimentation formelle et matérielle permettant de distinguer diverses stratégies qui «bouleversent nos habitudes de lecture » (13). Martin s'appuie sur une poignée d'exemples récurrents : les œuvres de Chris Ware (Jimmy Corrigan) et de Daniel Mark Z. Danielewski (House of Leaves) forment les véritables piliers de l'argumentation, à côté d'autres "œuvresexemples » telles que A Humument de Tom Phillips, Tree of Codes de Jonathan Safran Foer ou encore $S$. de Doug Dorst, autant de romans qui se caractérisent par leurs formats peu usuels. Martin convoque par ailleurs une série conséquente d'exemples complémentaires qui viennent ajuster et renforcer l'argumentation.

4 L'ouvrage se développe en trois temps, resserrant progressivement l'échelle d'analyse. La première partie considère le livre dans sa tridimensionnalité, comme objet matériel. Cette partie s'intéresse ainsi à la diversité des formats disponibles et à leur exploitation créative : le travail de Chris Ware est parlant à cet égard, car l'œuvre du dessinateur est justement marquée par une multiplicité de formats et un contrôle revendiqué du processus de production matériel. Le choix de formats autres ou l'exploitation inhabituelle de formats standardisés mènent ainsi à une prise de conscience chez le lecteur du livre comme objet matériel, tridimensionnel, manipulable. Martin étudie également le rôle crucial, mais flou, du paratexte pour de telles œuvres qui compliquent le «seuil» du paratexte en sapant les conventions éditoriales et en utilisant la couverture et les espaces marginaux pour déjà y situer une partie du récit. Cette importance du paratexte permet ainsi d'établir un contrat de lecture marqué au sceau de l'inhabituel.

5 La seconde partie se penche ensuite sur l'espace de la page et son organisation spatiale pluri-linéaire. Partant, Martin poursuit le fil directeur de la matérialité en soulignant la page imprimée comme ressource d'affects, de sensorialité, et potentiellement de jeux d'impressions qui sont motivés dans le récit multimodal, un aspect pourtant souvent ignoré des analyses et qui mène Martin à évoquer une série d'œuvres jouant sur la page " manipulée, malmenée et inattendue », pour reprendre le titre du quatrième chapitre: tourner la page, acte internalisé, devient alors un acte signifiant ou surprenant, dont les effets doivent être ménagés. C'est l'attention au rythme de lecture qui unit les différents chapitres de cette partie et Martin décrit avec précision la manière dont les auteurs varient leurs stratégies multimodales ou au contraire recréent de nouvelles habitudes chez le lecteur, qu'il faudra à nouveau déjouer. 
6 C'est enfin le signe typographique qui est au centre de l'analyse dans la dernière partie, nous menant des jeux de variations typographiques dans le roman multimodal à la visualité du texte dans la bande dessinée. À travers ces exemples, et à l'instar de la démarche générale de l'auteur, il s'agit de révéler l'importance des choix typographiques sur la base d'exemples qui mobilisent les ressources visuelles et textuelles à des fins narratives et poétiques. House of Leaves et Jimmy Corrigan sont là encore des exemples parfaits pour illustrer ces stratégies qui déjouent les rapports texte-image conventionnels.

7 Tout au long de l'ouvrage de Côme Martin, c'est donc l'expérience de lecture, en ce qu'elle est bousculée par une série de stratégies multimodales, qui est au premier plan de l'analyse. À partir d'une lecture minutieuse et parfois microscopique (comme l'analyse des moindres détails typographiques), l'ouvrage monte ainsi en généralités et propose une analyse narratologique plus transversale, qui a davantage de gains théoriques, mais place sur un second plan l'historicité des habitudes de lecture, liées aux formats de publication. L'ouvrage se penche sur un moment culturel particulier de la transformation du roman aux États-Unis, où l'institutionnalisation du graphic novel au sein de l'économie littéraire en a fait un objet éditorial occupant le même terrain que le roman multimodal et menant à de nombreuses hybridations et aller-retours ${ }^{2}$. Le statut d'auteurs comme Chris Ware et Mark Z. Danielewski, dont les œuvres expérimentales ont connu un succès surprenant, marqué par différentes formes de continuation et de réédition, fait bien état de cette transformation. Côme Martin aborde ces questions en creux et par petites touches au fil de l'ouvrage mais une mise en contexte plus systématique des habitudes de lecture, relative aux différents médias étudiés, aurait complété l'analyse fine des stratégies multimodales déployées.

Si la réflexion théorique sur les stratégies de lecture et leur perturbation prime sur leur cadre historique dans l'ouvrage de Côme Martin, c'est peut-être aussi que cette réflexion a vocation à être mise en pratique. Martin est par ailleurs auteur d'œuvres qui expérimentent avec le format, invitent le lecteur à jouer un rôle résolument actif dans l'assemblage narratif, où format, forme et fiction se répondent. En témoigne L'Envers $d u$ Dédale ${ }^{3}$, boîte-livre qui n'est pas sans rappeler Building Stories de Chris Ware, où chaque page est décousue et marquée d'un trait coloré, amenant à différentes combinaisons possibles et invitant le lecteur à un arpentage labyrinthique de l'univers fictionnel. Cette pratique d'écrivain se ressent implicitement tout au long de Lire le récit multimodal, un ouvrage toujours attentif au précis de la composition et de l'écriture comme contrainte et jeu avec des formats préexistants ou à inventer, et dont la prise en main par le lecteur doit toujours être pensée et réfléchie en amont, pour que s'établisse la "poétique du tangage, de la bousculade » (225) recherchée $e^{4}$.

\section{NOTES}

1. Voir notamment Alison Gibbons. Multimodality, Cognition, and Experimental Literature. Abingdon-on-Thames : Routledge, 2012 ; Wolfgang Hallet. « The Rise of the Multimodal 
Novel: Generic Change and Its Narratological Implications ». Storyworlds across Media: Towards a Media-Conscious Narratology. Dir. Marie-Laure Ryan \& Jan-Noël Thon. Nebraska : University of Nebraska Press, 2013. 151-172.

2. Sur l'institutionnalisation littéraire du roman graphique et sur les questions que celui-ci pose au roman littéraire dans son rapport à l'image, voir Jan Baetens et Hugo Frey. «The Graphic Novel ». The Cambridge Companion to the Novel. Dir. Eric Bulson. New York; Cambridge : Cambridge University Press, 2018. 238-253.

3. Côme Martin. L'Envers du dédale. Angoulême : Éditions Rutabaga, 2018.

4. Par souci de transparence et pour souligner tout potentiel conflit d'intérêt, il convient d'indiquer que l'auteur du compte-rendu est affilié au groupe ACME, dont les membres participent au suivi éditorial de la collection éponyme aux Presses universitaires de Liège. L'auteur n'a cependant pas été impliqué dans la procédure d'évaluation et de publication de ce volume.

\section{AUTEURS}

\section{BENOÎT CRUCIFIX}

Université de Gand 